This article has been downloaded from IOPscience. Please scroll down to see the full text article. (http://iopscience.iop.org/1126-6708/2007/10/109)

More related content is available

Download details:

IP Address: 137.138.125.160

The article was downloaded on 12/12/2007 at 14:04

Please note that terms and conditions apply. 


\section{Precision electroweak calculation of the production of a high transverse-momentum lepton pair at hadron colliders}

\section{Carlo M. Carloni Calame}

CERN, Physics Department, TH Unit,

1211 Geneva, Switzerland

E-mail: Carlo.Carloni.Calame@pv.infn.it

\section{Guido Montagna}

Dipartimento di Fisica Nucleare e Teorica, Università di Pavia and

INFN, Sezione di Pavia,

Via A.Bassi 6, I-27100 Pavia, Italy

E-mail: Guido.Montagna@pv.infn.it

\section{Oreste Nicrosini}

INFN, Sezione di Pavia,

Via A.Bassi 6, I-27100 Pavia, Italy

E-mail: Oreste.Nicrosini@pv.infn.it

\section{Alessandro Vicini}

Dipartimento di Fisica, Università degli Studi di Milano and

INFN, Sezione di Milano,

Via Celoria 16, I-20133 Milano, Italy

E-mail: Alessandro.Vicini@mi.infn.it

ABSTRACT: We present a detailed study of the production of a high transverse-momentum lepton pair at hadron colliders, which includes the exact $\mathcal{O}(\alpha)$ electroweak corrections properly matched with leading logarithmic effects due to multiple photon emission, as required by the experiments at the Fermilab Tevatron and the CERN LHC. Numerical results for the relevant observables of single $Z$-boson production at hadron colliders are presented. The impact of the radiative corrections is discussed in detail. The presence in the proton of a photon density is considered and the effects of the photon-induced partonic subprocesses are analyzed. The calculation has been implemented in the new version of the event generator HORACE, which is available for precision simulations of the neutral and charged current Drell-Yan processes.

Keywords: Standard Model, Hadronic Colliders. 


\section{Contents}

1. Introduction 1

2. Partonic process: matrix elements calculation 3

2.1 Born approximation 3

2.2 The $\mathcal{O}(\alpha)$ calculation

2.2.1 Virtual corrections

2.2.2 Bremsstrahlung corrections 8

2.2.3 Photon-induced processes 9

2.3 Higher-order electroweak effects 9

3. Matching QED higher orders and hadron-level cross section 10

3.1 Matching 10

3.2 Hadron-level cross section 10

4. Numerical results 12

5. Conclusions 18

\section{Introduction}

At hadron colliders, such as the Fermilab Tevatron and the CERN LHC, the production of a high transverse-momentum lepton pair, known as Drell-Yan process [1], plays an important role: it allows, in the neutral current channel, to study the physics of the $Z$ boson and, in particular, to determine the effective weak mixing angle from the measurement of the forward-backward asymmetry [2]; in the charged current channel, a high precision determination of two fundamental parameters of the Standard Model, namely the mass and the decay width of the $W$ boson, can be obtained [3]; it provides, both in the neutral and charged current channel, stringent constraints on the density functions which describe the partonic content of the proton [4]; it can be used as a standard reference process and, therefore, as a luminosity monitor of the collider [5, 6]. Furthermore, it represents a background to the search for new heavy gauge bosons [7].

The accuracy in the determination of the theoretical cross section has greatly increased over the years. The calculation of next-to-leading order (NLO) QCD corrections 8 has been one of the first test grounds of perturbative QCD. Next-to-next-to-leading order (NNLO) QCD corrections to the total cross section have been computed in ref. [9], but differential distributions with the same accuracy have been obtained only recently in ref. [10]. The size of the NNLO QCD corrections and the improved stability of the results against 
variations of the renormalization/factorization scales raises the question of the relevance of the $\mathcal{O}(\alpha)$ electroweak (EW) radiative corrections, which were computed, in the neutral current channel, first considering the gauge-invariant subset of QED corrections [1] and then the complete set of $\mathcal{O}(\alpha)$ EW corrections [12, 13].

A realistic phenomenological study and the data analysis require the inclusion of the relevant radiative corrections and their implementation into Monte Carlo event generators, in order to simulate all the experimental cuts and to allow, for instance, an accurate determination of the detector acceptances. The Drell-Yan processes are included in the standard QCD Parton Shower generators HERWIG [14] and PYTHIA [15]. Recently, there has been important progress to improve the QCD radiation description to NLO, which has been implemented in the code MC@NLO [16]. Another important issue is a reliable description of the intrinsic transverse momentum of the gauge bosons, which can be obtained by resumming up to all orders contributions due to multiple soft-gluon radiation. The generator RESBOS [17], used for data analysis at Tevatron, includes these effects.

If the inclusion of QCD radiation is mandatory for the simulation of any process at hadron colliders, one should not neglect the impact of EW corrections on the precision measurement of some Standard Model (SM) observables, like the $Z$ boson mass and decay width, important for detector calibration. For instance, the generator ZGRAD [11, 12] includes the exact $\mathcal{O}(\alpha)$ EW corrections, which have been shown to induce a shift on the value of $m_{Z}$ extracted from the Tevatron data of few hundreds of $\mathrm{MeV}$ [3], depending on the experimental set up, mostly due to final-state QED radiation. Some event generators can also account for multiple-photon radiation: in the published version of HORACE [18-20] QED radiation in leading-log approximation was simulated by means of a QED Parton Shower [21]; the standard tool PHOTOS [22] can be adopted to describe QED radiation in the $Z$ decay.

Since the Drell-Yan events can be used, in principle, to determine the collider luminosity at a few per cent level, the theoretical cross section must be known with the same accuracy, requiring also the inclusion of $\mathcal{O}(\alpha)$ EW corrections. Furthermore, the $\mathcal{O}(\alpha)$ EW contributions give large corrections to the high tails of the invariant mass and lepton transverse momentum distributions, because of the presence of large EW Sudakov logarithms [12], thus affecting significantly the SM normalization in the search for new heavy gauge bosons.

Neutral and charged current Drell-Yan processes can be combined to obtain an independent way of measuring the $W$ boson mass: in fact, it has been shown [23] that the ratios of $W$ and $Z$ observables, properly defined, is less sensitive to missing higher-order QCD effects and to the $p d f$ uncertainties; on the other hand, these ratios are sensitive to the value of the $W$ mass and can be exploited for its precise determination. The impact of the EW corrections in this framework is relevant. Preliminary studies [24] have shown that these corrections do not cancel in the ratio and could play an important role in the $W$ mass determination according to this method. It is therefore very important to have a tool to perform a realistic simulation of the Drell-Yan processes, including $\mathcal{O}(\alpha)$ and multiple photon corrections, not only in the charged current channel [20], but also in the neutral current case. 
The aim of this paper is to present a precision EW calculation of the neutral current Drell-Yan process, which includes the exact $\mathcal{O}(\alpha)$ EW matrix elements properly matched with leading logarithmic higher-order QED corrections in the Parton Shower approach. The matching of perturbative corrections with Parton Shower, which is a topic of great interest in modern QCD simulations [25], has already been illustrated in ref. [20] and is realized along the lines already presented in ref. [26]. The use of the $p d f$ set MRST2004QED [27], which describes the partonic content of the proton also in terms of a photon density, implies the calculation of photon-induced partonic processes. The latter contribute to the inclusive cross section $\sigma\left(p \stackrel{(-)}{\rightarrow} \rightarrow l^{+} l^{-}(n \gamma)+X\right)$ and are of the same perturbative order as the Born Drell-Yan tree-level reaction (subprocess $\gamma \gamma \rightarrow l^{+} l^{-}$) or of the same perturbative order of the $\mathcal{O}(\alpha)$ corrections (subprocess $\gamma q \rightarrow l^{+} l^{-} q$ ). The latter have been recently addressed to in ref. 28].

Several distributions of physical interest are analyzed, disentangling the effect of different classes of radiative corrections and discussing various sources of theoretical uncertainty. The calculation is implemented in the new version of the Monte Carlo event generator HORACE, ${ }^{1}$ which combines, in a unique tool, the good features of the QED Parton Shower approach with the additional effects present in the exact $\mathcal{O}(\alpha)$ EW calculation. This task is non trivial from several technical points of view and faces all the conceptual problems of developing a NLO event generator.

The paper is organized as follows. In section 2 we present the calculation of the partonic subprocesses and of $\mathcal{O}(\alpha)$ radiative corrections. In section 3.1 we describe the matching of the fixed-order results with the QED Parton Shower. In section 3.2 we present the computation of the hadron-level cross section $\left.\sigma(p) \rightarrow l^{+} l^{-}(n \gamma)+X\right)$ and discuss the subtraction of the initial-state collinear singularities to all orders. In section 1 we present phenomenological results for several physical distributions and discuss the impact of $\mathcal{O}(\alpha)$ EW corrections, photon-induced processes and higher-order QED contributions. Finally, in section 5 we draw our conclusions and discuss possible developments of this work.

\section{Partonic process: matrix elements calculation}

We consider a description of the proton which includes also the presence of a photon as a parton, described by the corresponding density function, as done for instance in ref. [27]. The lowest-order cross section for the production of a high transverse-momentum lepton pair starts at $\mathcal{O}\left(\alpha^{2}\right)$ and receives contributions from two partonic subprocesses, namely $q\left(p_{1}\right) \bar{q}\left(p_{2}\right) \rightarrow l^{-}\left(p_{3}\right) l^{+}\left(p_{4}\right)$ and also $\gamma\left(p_{1}\right) \gamma\left(p_{2}\right) \rightarrow l^{-}\left(p_{3}\right) l^{+}\left(p_{4}\right)$. When considering this final state at $\mathcal{O}\left(\alpha^{3}\right)$, we must include, in addition to the usual radiative process $q\left(p_{1}\right) \bar{q}\left(p_{2}\right) \rightarrow$ $l^{-}\left(p_{3}\right) l^{+}\left(p_{4}\right) \gamma\left(p_{5}\right)$, also a new subprocess, i.e. $q\left(p_{1}\right) \gamma\left(p_{2}\right) \rightarrow l^{-}\left(p_{3}\right) l^{+}\left(p_{4}\right) q\left(p_{5}\right)$, which contributes to the inclusive signature.

\subsection{Born approximation}

We start considering the neutral current Drell-Yan partonic process $q\left(p_{1}\right) \bar{q}\left(p_{2}\right) \rightarrow$

\footnotetext{
${ }^{1}$ The code can be downloaded from the url http://www.pv.infn.it/hepcomplex/horace.htm
} 


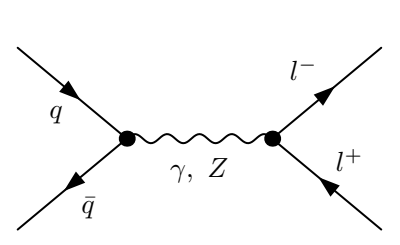

(a)

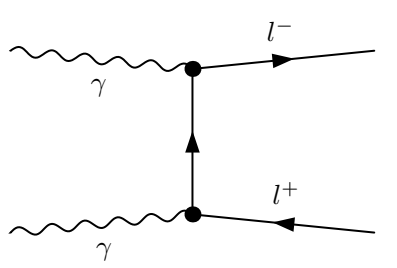

(b)

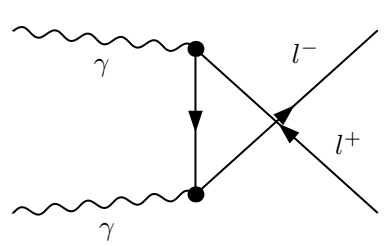

(c)

Figure 1: Born diagrams for the $q \bar{q}(\mathrm{a})$ and for the $\gamma \gamma(\mathrm{b}, \mathrm{c})$ subprocesses.

$l^{-}\left(p_{3}\right) l^{+}\left(p_{4}\right)$, which is depicted in figure 1 (a). This process is a neutral current process and its amplitude, neglecting the Higgs-boson contribution, is mediated by $s$-channel photon and $Z$-boson exchange. In the unitary gauge, the tree-level amplitude reads as

$$
\begin{aligned}
\mathcal{M}_{0} & =\mathcal{M}_{\gamma}+\mathcal{M}_{Z} \\
\mathcal{M}_{\gamma} & =-e^{2} Q_{q} Q_{l} \frac{g_{\mu \nu}-k_{\mu} k_{\nu} / s}{s}\left[\bar{v}\left(p_{2}\right) \gamma^{\mu} u\left(p_{1}\right)\right]\left[\bar{u}\left(p_{3}\right) \gamma^{\nu} v\left(p_{4}\right)\right] \\
& \equiv-e^{2} Q_{q} Q_{l} \frac{g_{\mu \nu}-k_{\mu} k_{\nu} / s}{s} J_{\mathrm{em}}^{\mu} J_{\mathrm{em}}^{\nu} \\
\mathcal{M}_{Z} & =-\frac{e^{2}}{s_{\theta}^{2} c_{\theta}^{2}} \frac{g_{\mu \nu}-k_{\mu} k_{\nu} / s}{s-m_{Z}^{2}+i \Gamma_{Z} m_{Z}}\left[\bar{v}\left(p_{2}\right)\left(v_{q} \gamma^{\mu}+a_{q} \gamma^{\mu} \gamma_{5}\right) u\left(p_{1}\right)\right]\left[\bar{u}\left(p_{3}\right)\left(v_{l} \gamma^{\nu}+a_{l} \gamma^{\nu} \gamma_{5}\right) v\left(p_{4}\right)\right] \\
& \equiv-\frac{e^{2}}{s_{\theta}^{2} c_{\theta}^{2}} \frac{g_{\mu \nu}-k_{\mu} k_{\nu} / s}{s-m_{Z}^{2}+i \Gamma_{Z} m_{Z}} J_{Z, q \bar{q}}^{\mu} J_{Z, l^{+} l^{-}}^{\nu}
\end{aligned}
$$

where $m_{Z}$ is the $Z$-boson mass and $\Gamma_{Z}$ is the $Z$ decay width, necessary to describe the $Z$ resonance region, $s=\left(p_{1}+p_{2}\right)^{2}$ is the squared partonic center-of-mass (c.m.) energy and $k^{\mu}=p_{1}^{\mu}+p_{2}^{\mu}, \alpha=e^{2} /(4 \pi)$ is the fine structure constant, $c_{\theta} \equiv m_{W} / m_{Z}$ is the cosine of the weak mixing angle. The vector and axial-vector couplings of the $Z$-boson to fermions are $v_{f}=T_{f}-2 Q_{f} s_{\theta}^{2}$ and $a_{f}=-T_{f}$ where $T_{f}= \pm 1 / 2$ is the third component of the weak isospin and $Q_{f}$ is the electric charge of the fermion $f$.

The subprocess $\gamma\left(p_{1}\right) \gamma\left(p_{2}\right) \rightarrow l^{-}\left(p_{3}\right) l^{+}\left(p_{4}\right)$, which is depicted in figure 1 (b,c), is, at lowest order, a pure QED reaction, whose differential cross section, in the partonic c.m. frame and neglecting all fermion masses, reads as

$$
\frac{d \hat{\sigma}_{\gamma \gamma}}{d \cos \theta}=\frac{2 \pi \alpha^{2}}{s}\left(\frac{1+\cos ^{2} \theta}{\sin ^{2} \theta}\right)
$$

\subsection{The $\mathcal{O}(\alpha)$ calculation}

The complete $\mathcal{O}(\alpha)$ EW corrections to the neutral current Drell-Yan process have already been computed in refs. 12, 13]. We have repeated independently the calculation and included in addition the photon-induced processes. We summarize here the main features of our approach.

The $\mathcal{O}(\alpha)$ corrections include the contribution of real and virtual corrections. The virtual corrections follow from the perturbative expansion of the $2 \rightarrow 2$ scattering amplitude 


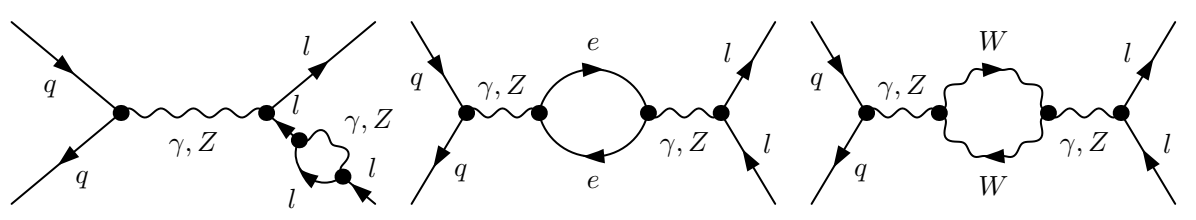

(a)

(b)

(c)

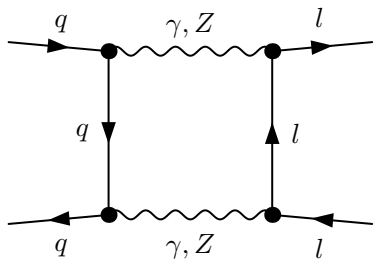

$(d)$

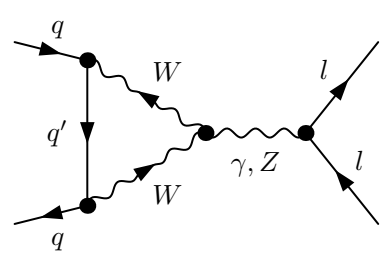

(e)

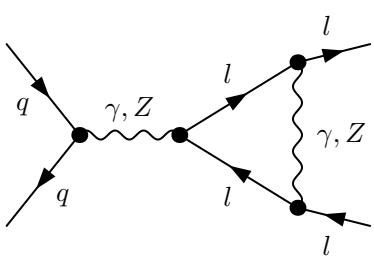

$(f)$

Figure 2: Some examples of one-loop virtual diagrams.

$\mathcal{M}=\mathcal{M}_{0}+\mathcal{M}_{\alpha}^{\text {virt }}+\cdots$ and contribute, at $\mathcal{O}(\alpha)$, with $2 \operatorname{Re}\left(\mathcal{M}_{\alpha}^{\text {virt }} \mathcal{M}_{0}^{*}\right)$. The $\mathcal{O}(\alpha)$ virtual amplitude includes two contributions, namely the one-loop renormalization of the treelevel amplitude and the virtual one-loop diagrams. The real corrections are due to the emission of one extra real photon and represent the lowest order of the radiative process $q\left(p_{1}\right) \bar{q}\left(p_{2}\right) \rightarrow l^{-}\left(p_{3}\right) l^{+}\left(p_{4}\right) \gamma(k)$. They can be further divided into soft and hard corrections, $\mathcal{M}_{1}=\mathcal{M}_{1}^{\text {soft }}+\mathcal{M}_{1}^{\text {hard }}$. The former satisfies, by definition, the Born-like $2 \rightarrow 2$ kinematics and can be factorized as $\left|\mathcal{M}_{1}^{\text {soft }}\right|^{2}=\delta_{\mathrm{SB}}\left|\mathcal{M}_{0}\right|^{2}$, where $\delta_{\mathrm{SB}}$ is a universal factor that depends only on the properties of the external particles. The total cross section includes soft and hard corrections and is independent of the cut-off used to define the two energy regions. Virtual and real soft corrections are separately divergent due to the emission of soft photons, but the divergence cancels in the sum of the two contributions.

\subsubsection{Virtual corrections}

The $\mathcal{O}(\alpha)$ virtual corrections to a $2 \rightarrow 2$ reaction include the contribution of counterterms, self-energy, vertex and box corrections. Few diagrams representative of the different kinds of corrections are depicted in figure 2. The $\mathcal{O}(\alpha)$ virtual corrections have been calculated using the packages FeynArts and FormCalc [29, 30]. The numerical evaluation of the 1-loop integrals has been done using the package LoopTools2 [30], based on the library ff [31]. We will write the 1 -loop virtual amplitude as $\mathcal{M}_{\alpha}^{\text {virt }}=\mathcal{M}_{\alpha}^{\text {cts }}+\mathcal{M}_{\alpha}^{\text {self }}+\mathcal{M}_{\alpha}^{\text {vertex }}+\mathcal{M}_{\alpha}^{\text {box }}$, where $\mathcal{M}_{\alpha}^{\text {cts }}$ includes all the counterterms and the wave function corrections on the external legs, $\mathcal{M}_{\alpha}^{\text {self }}$ describes the self-energy corrections to the photon and to the $Z$ propagator and the contribution due to the $\gamma-Z$ mixing and $\mathcal{M}_{\alpha}^{\text {vertex,box }}$ describe respectively the vertex and the box corrections. The mass of the fermions in the scalar 1-loop integrals regularizes in a natural way the mass singularities due to the emission of a (virtual) collinear photon. The infrared divergence of the integrals has been regularized by means of a small photon mass $\lambda$. 
The introduction of the $Z$ decay width in the propagator of the $Z$ boson is mandatory to describe the resonance region and to regularize the divergence due to the pole of the propagator. In the 't Hooft-Feynman gauge, the 1-loop corrections to the $Z$ propagator read

$$
\left(-i g^{\mu \nu}\right) \frac{1}{s-m_{Z}^{2}+i \Gamma_{Z} m_{Z}}\left(\operatorname{Re}\left(\Pi_{\mathrm{ZZ}}(s)\right)+\delta m_{Z}^{2}+\left(s-m_{Z}^{2}\right) \delta Z_{Z}\right) \frac{1}{s-m_{Z}^{2}}
$$

where $\Pi_{\mathrm{ZZ}}(s)$ is the transverse part of the $Z$ self-energy corrections, $\delta m_{Z}^{2}$ and $\delta Z_{Z}$ are the $Z$ mass and wave function renormalization constants, respectively. The two counterterms cancel the divergences present in the self-energy corrections. We remark that we have to take the real part of $\Pi_{\mathrm{ZZ}}(s)$ in order to avoid a double counting with the decay width in the denominator. In fact the $\mathcal{O}(\alpha)$ contribution is $2 \operatorname{Re}\left(\mathcal{M}_{\mathrm{ZZ}}^{\text {self }} \mathcal{M}_{0}^{*}\right)$ : the real part prescription in the latter expression avoids the double counting in the interference with the $Z$ treelevel diagram, but not the one in the interference with the photon tree-level diagram. Furthermore, the second factor $1 /\left(s-m_{Z}^{2}\right)$ in eq. (2.3) is not corrected by the decay width, again to avoid a double counting; we can check, by expanding the self-energy corrections around $s=m_{Z}^{2}$, that the $\mathcal{O}(\alpha)$ expression is regular for $s \rightarrow m_{Z}^{2}$.

The $\gamma-Z$ mixing diagrams require the introduction in the $Z$ propagator of a decay width to describe the $Z$-resonance region, i.e.

$$
\left(-i g^{\mu \nu}\right) \frac{1}{s-m_{Z}^{2}+i \Gamma_{Z} m_{Z}}\left(\operatorname{Re}\left(\Pi_{\gamma Z}(s)\right)+\frac{\left(s-m_{Z}^{2}\right)}{2} \delta Z_{\gamma Z}+\frac{1}{2} \delta Z_{Z \gamma}\right) \frac{1}{s}
$$

Also in this case we need to take the real part of $\Pi_{\gamma Z}$ to avoid the double counting between the imaginary part of the self-energy and the imaginary term proportional to the decay width.

Examples of vertex and box corrections are depicted in figure 2. The abelian vertex diagrams and the box diagrams with a photonic correction are infrared divergent. All the vertex and box diagrams with a $Z$-boson exchange yield the so-called EW Sudakov logarithms, namely terms like $\alpha \log ^{2}\left(s / m_{Z}^{2}\right)$, whose importance grows for large invariant mass of the final-state lepton pair, while they are almost negligible at the $Z$ resonance. The box diagrams with a photon and a $Z$ boson in the loop yield a logarithmic divergence at $s=m_{Z}^{2}$. Since all the terms with $\log \left(s-m_{Z}^{2}\right)$ form a gauge invariant subset of corrections, we regularized the divergence by replacing everywhere $\log \left(s-m_{Z}^{2}\right) \rightarrow \log \left(s-m_{Z}^{2}+i \Gamma_{Z} m_{Z}\right)$. The $\gamma Z$ boxes are resonant at $s \sim m_{Z}^{2}$. The divergence has been regularized by modifying the box amplitude in the following way

$$
\begin{aligned}
\mathcal{M}^{\text {box }} & =\mathcal{M}_{\gamma \gamma}^{\text {box }}+\mathcal{M}_{\gamma Z}^{\text {box }}+\mathcal{M}_{Z Z}^{\text {box }} \rightarrow \\
& \rightarrow \mathcal{M}_{\gamma \gamma}^{\text {box }}+\frac{s-m_{Z}^{2}}{s-m_{Z}^{2}+i \Gamma_{Z} m_{Z}} \mathcal{M}_{\gamma Z}^{\text {box }}+\mathcal{M}_{Z Z}^{\text {box }}
\end{aligned}
$$

The calculation has been repeated with two different gauge choices, namely the $R_{\xi}$ with $\xi=1$ gauge and the background field gauge, with parameter $q=1$ 32]. The two results perfectly agree, and this is an important check of the calculation of the bosonic self-energy and of the non-abelian vertex corrections. 
Concerning the renormalization of the 1-loop amplitude, the ultraviolet divergences which appear from the virtual diagrams can be cancelled with the mass, $\delta m_{Z}^{2}$, and wave function, $\delta Z_{Z}$, renormalization constants of the $Z$ boson, with the wave function renormalization of the photon, $\delta Z_{\gamma \gamma}$, and of the $\gamma Z$ mixing, $\delta Z_{\gamma z}$ and $\delta Z_{Z \gamma}$ and by the renormalization of the two vertices $Z q \bar{q}$ and $Z l^{+} l^{-}$. The latter include the charge renormalization and the wave function renormalization of the external fermions, of the photon and of the $Z$ boson. We use the electric charge and the gauge boson masses $e, m_{W}, m_{Z}$ as input parameters, we replace the bare coupling $e_{0}$ in terms of the renormalized quantity and of the corresponding counterterm $e_{0}=e(1-\delta e / e)$. The electric charge counterterm is fixed by the request that in Thomson scattering the renormalized charge is given by the fine structure constant; its expression depends on the quark masses running in the photon vacuum polarization, the value of which can be adjusted in order to make the running electric charge reproduces the value $\alpha\left(m_{z}^{2}\right)$ [33]. For the sake of brevity, we can introduce a counterterm for the weak mixing angle, which is given by a combination of the mass counterterms of the $W$ and $Z$ bosons, following from its definition. The $W$ - and $Z$-boson mass and wave function renormalization constants, the $\gamma \gamma$ and $\gamma Z$ wave function renormalization constants are defined in the on-shell scheme.

The choice of the input parameters of the SM lagrangian has a numerical impact on the calculation of the physical observables. In order to obtain predictions with the smallest possible parametric uncertainty, it would be convenient to choose as input parameters $\alpha, G_{\mu}, m_{Z}$, which was the common choice at LEP1. The drawback is that in this approach $m_{W}$ is a prediction of the SM, whereas, at hadron colliders, it would be interesting to keep it as an input, which can be measured fitting the data. It is therefore more useful to choose $\alpha, m_{W}, m_{Z}$ as inputs. For instance, the method proposed in ref. 23] to study the ratio of $W$ and $Z$ observables and to use it to determine the $W$ mass requires $m_{W}$ as input of both charged and neutral current simulations, which will then be compared with the data.

Unfortunately, the choice $\alpha, m_{W}, m_{Z}$ suffers, if strictly applied, of the ambiguity due to the choice of the quark mass values in the photon vacuum polarization. The ambiguity can be removed by re-expressing, in the Born amplitude, the fine structure constant in terms of a different quantity, like the Fermi constant $G_{\mu}$ or the effective electromagnetic coupling at the scale $q^{2}, \alpha\left(q^{2}\right)$, whose $\mathcal{O}(\alpha)$ expressions exactly cancel the quark mass dependence due to the vacuum polarization diagrams. In order to rewrite the tree-level amplitude in terms of these new couplings, we follow the prescriptions developed at LEP1 to study the $Z$-resonance and treat separately the photon- and the $Z$-diagram contributions. In $\mathcal{M}_{\gamma}$ we replace

$$
\begin{aligned}
e^{2} \rightarrow e^{2}\left(q^{2}\right) & =e^{2} /\left(1-\Delta \alpha\left(q^{2}\right)\right) \\
\Delta \alpha\left(q^{2}\right) & =\Pi_{\gamma \gamma}^{(f)}\left(q^{2}\right)-\Pi_{\gamma \gamma}^{(f)}(0)
\end{aligned}
$$

where $\Delta \alpha\left(q^{2}\right)$ expresses the running of the electric charge due to the fermionic photon vacuum polarization $\left(\Pi_{\gamma \gamma}^{(f)}\left(q^{2}\right)\right.$ is related to the transverse part of the photon self-energy by $\left.A_{\gamma \gamma}^{(f)}\left(q^{2}\right)=q^{2} \Pi_{\gamma \gamma}^{(f)}\left(q^{2}\right)\right)$. The quantity $\Delta \alpha$ includes also the non-perturbative hadronic contribution due to a loop of quarks at low virtualities. For the evaluation of $\Delta \alpha_{\text {had }}^{(5)}$ we 


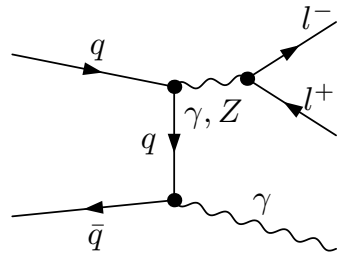

(a)

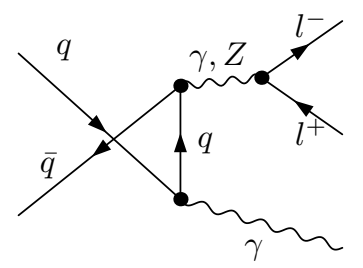

$(b)$

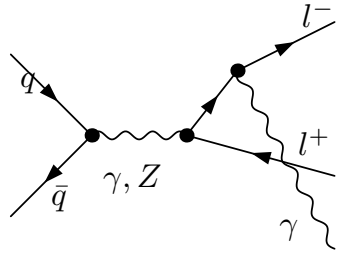

$(c)$

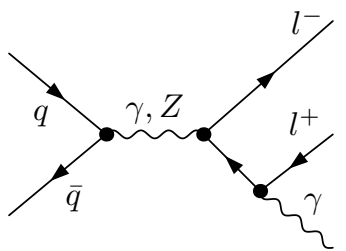

$(d)$

Figure 3: $\mathcal{O}(\alpha)$ bremsstrahlung diagrams.

use the routine hadr $5 \mathrm{n}$, fred. Because we include the photon vacuum polarization effects in the lowest-order coupling, we have to subtract the $\mathcal{O}(\alpha)$ expansion of $e^{2}\left(q^{2}\right)$, to avoid a double counting when we include the full set of $\mathcal{O}(\alpha)$ corrections.

In the case of $\mathcal{M}_{Z}$ we can rewrite $e^{2} /\left(s_{\theta}^{2} c_{\theta}^{2}\right)$ as $g^{2} / c_{\theta}^{2}$ and then use the relation, computed up to $\mathcal{O}(\alpha)$, of the weak coupling $g$ with the Fermi constant and the $W$-boson mass

$$
\frac{G_{\mu}}{\sqrt{2}}=\frac{g^{2}}{8 m_{W}^{2}}(1+\Delta r)
$$

The quantity $\Delta r$ represents all the radiative corrections to the muon-decay amplitude [34.

\subsubsection{Bremsstrahlung corrections}

The real radiative corrections to the neutral current Drell-Yan process, described by the amplitude $\mathcal{M}_{1}$, are given by all the Feynman diagrams (figure 3) with the emission of one extra photon from all the electrically charged legs of the Born diagrams.

The probability amplitude has been calculated in the unitary gauge with massive fermions. We integrate the squared matrix element over the whole photon phase space and split the allowed photon energy range in two intervals, $[\lambda, \Delta E]$ and $\left[\Delta E, E_{\max }\right]$. The cut-off $\Delta E \ll \sqrt{s}$ is chosen in such a way that the photon with smaller energy is considered soft and does not modify the $2 \rightarrow 2$ kinematics of the Born amplitude. The small photon mass $\lambda$ has been introduced to regularize the infrared divergence. In this energy region the phase space integral, including the full angular integration, can be solved analytically. The result can be expressed in a factorized form, as

$$
\int_{\Omega} \frac{d^{3} \mathbf{k}_{\gamma}}{(2 \pi)^{3} 2 E_{\gamma}}\left|\mathcal{M}_{1}\right|^{2}=\left|\mathcal{M}_{0}\right|^{2} \sum_{f=q, \bar{q}, l^{+} l^{-}} \delta_{\mathrm{SB}}(f, \lambda)
$$

where the soft Bremsstrahlung factor, see e.g. ref. [35], depends on the mass and electric charge of the external radiating particles and the phase-space region $\Omega$ is defined by the request that the photon energy $E_{\gamma}$ satisfies $\lambda \leq E_{\gamma} \leq \Delta E$. We have explicitly checked that the sum of the virtual and soft-real contributions is independent of the choice of the photon mass $\lambda$, in the limit of small $\lambda$ values.

In the hard energy region the phase-space integration has been performed numerically, with Monte Carlo techniques improved by importance sampling to take care of collinear 


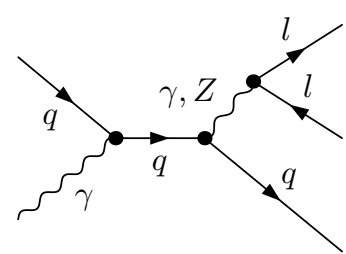

$(a)$

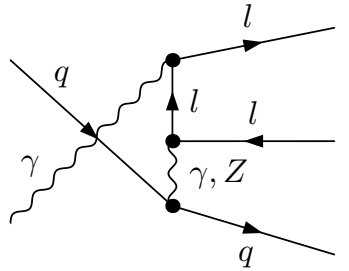

(b)

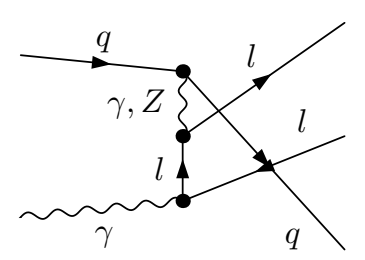

$(c)$

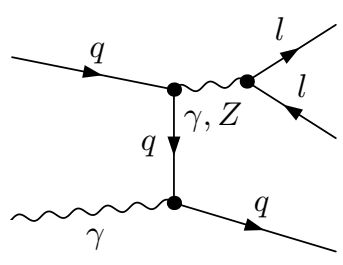

$(d)$

Figure 4: Photon-induced process diagrams.

and infrared singularities, as well as the peaking behaviour around the $Z$ resonance. The sum of the soft and of the hard photon cross sections is independent of the cut-off $\Delta E$. We have checked the independence of our numerical results from the choice of the infrared separator $\varepsilon \equiv \Delta E / E$ for $10^{-8} \leq \varepsilon \leq 10^{-4}$.

\subsubsection{Photon-induced processes}

In ref. 27 it has been proposed a new parametrization of the partonic content of the proton, which also includes a photon probability density. When using this set of $p d f$, the inclusive cross section $\sigma\left(p \stackrel{(-)}{p} \rightarrow l^{+} l^{-}+X\right)$ receives contributions also from the partonic subprocesses $q\left(p_{1}\right) \gamma\left(p_{2}\right) \rightarrow l^{+}\left(p_{3}\right) l^{-}\left(p_{4}\right) q(k)$ (photon-induced), depicted in figure 1 . The latter are of the same perturbative order as the real bremsstrahlung corrections described in the previous subsection, i.e. they are an $\mathcal{O}(\alpha)$ correction to the Born process of eq. (2.1). The squared amplitude of the photon-induced processes can be obtained by crossing symmetry from the real bremsstrahlung one, evaluating the latter with the exchange $\left(p_{2} \leftrightarrow-k\right)$ and multiplying the result by a $(-1)$ factor to account for the exchange of a fermionic line.

\subsection{Higher-order electroweak effects}

To incorporate higher-order EW corrections in a Born-like expression written with effective couplings, we followed the approach of ref. [36], where the tree-level amplitude has been improved and takes into account all the self-energy and vertex corrections. The latter have been included by defining an effective overall coupling and an effective weak mixing angle.

The amplitude $\mathcal{M}_{Z}$ becomes

$$
\mathcal{M}_{Z}=\frac{i 8 G_{\mu} m_{Z}^{2}}{\sqrt{2}} \frac{\rho_{\mathrm{fi}}\left(q^{2}\right)}{1-\delta \rho_{\mathrm{irr}}} \frac{J_{Z, q \bar{q}} \cdot J_{Z, l^{+} l^{-}}}{q^{2}-m_{Z}^{2}+i \Gamma_{Z} m_{Z}}
$$

where the coupling $v_{f}$ of eq. (2.1) is replaced by $\tilde{v}_{f}=T_{f}-2 Q_{f} \kappa_{f}\left(q^{2}\right) s_{\theta}^{2}$. The definition of the quantities $\rho_{\mathrm{fi}}, \delta \rho_{\mathrm{irr}}, \kappa_{f}\left(q^{2}\right)$ can be found in ref. [36]. Eq. (2.9) incorporates also higherorder effects beyond $\mathcal{O}(\alpha)$, because of the resummation of $\delta \rho_{\text {irr }}$ and of the fermionic part of the $Z$ self-energy contained in $\rho_{\mathrm{fi}}$. Furthermore, $\delta \rho_{\text {irr }}=\delta \rho_{\text {irr }}^{(1)}+\delta \rho_{\text {irr }}^{(2)}$ contains also leading two-loop corrections. In the amplitude $\mathcal{M}_{\gamma}$ we replace the fine structure constant with the running electromagnetic coupling according to eq. (2.6). 
For the numerical analysis we combine the exact $\mathcal{O}(\alpha)$ corrections described in the previous subsections and evaluated with $\alpha(0), m_{W}, m_{Z}$ as input parameters, with the Bornlike expressions of this section. In order to avoid a double counting in the combination, we subtract the $\mathcal{O}(\alpha)$ content of eq. (2.9). All the numerical results shown in the following are obtained according to the EW scheme here described.

\section{Matching QED higher orders and hadron-level cross section}

\subsection{Matching}

In this section we describe the matching of the $\mathcal{O}(\alpha)$ EW calculation with higher-order QED corrections (cfr. ref. [26]). The latter can be included in a generic scattering cross section in the QED Parton Shower approach, which resums to all orders the leading logarithmic effects. At $\mathcal{O}(\alpha)$ the Parton Shower reproduces only the QED leading-log approximation of the exact $\mathcal{O}(\alpha)$ EW calculation presented in section 2. We would like to combine the exact $\mathcal{O}(\alpha)$ results and QED higher orders, to improve the approximation intrinsic to the Parton Shower, avoiding at the same time double counting at $\mathcal{O}(\alpha)$. A detailed description of the matching procedure can be found in refs. [20, 26]. Here we recall the basic ideas and results.

Our master formula is

$$
d \sigma^{\infty}=F_{\mathrm{SV}} \Pi\left(Q^{2}, \varepsilon\right) \sum_{n=0}^{\infty} \frac{1}{n !}\left(\prod_{i=0}^{n} F_{H, i}\right)\left|\mathcal{M}_{n, L L}\right|^{2} d \Phi_{n}
$$

and can be understood in the following way: 1) the tree-level cross-section ( $n=0$ in the sum) is corrected, in leading-log approximation, to all orders by the Sudakov form factor $\Pi\left(Q^{2}, \varepsilon\right)$, which accounts for virtual and soft-photon emission up to a scale $\varepsilon$ in a hard-scattering process characterized by a virtuality scale $Q ; 2)$ the resulting expression is dressed by the QED Parton Shower, with the real bremsstrahlung $n$-photons squared matrix elements in leading-log approximation; 3 ) the correction factors $F_{\mathrm{SV}}$ and $F_{H, i}$ provide the remaining $\mathcal{O}(\alpha)$ corrections missing in the leading-log approximation: in particular, $F_{\mathrm{SV}}$ contains the remainder of the soft plus virtual corrections, whereas $F_{H, i}$ gives, for the real photon emission, the correction due to the exact bremsstrahlung matrix element with respect to the Parton Shower approximation.

The expansion at $\mathcal{O}(\alpha)$ of eq. (3.1) coincides with the exact NLO cross section. Furthermore, all higher-order leading-log contributions are the same as in a pure QED Parton Shower. It is worth noticing that $F_{\mathrm{SV}}, F_{H, i}$ are, by construction, infrared safe and free of collinear logarithms.

\subsection{Hadron-level cross section}

In this section we discuss the calculation of the hadron-level cross section $\sigma(p p) \rightarrow l^{+} l^{-}+$ $n \gamma+X)$ and the procedure to subtract the initial-state mass singularities. This procedure makes the resulting hadron-level cross section independent of the (fictitious) value of the initial-state quark masses. 
The subtraction at $\mathcal{O}(\alpha)$ has been discussed, for example, in refs. [37, 38] and is obtained by a redefinition of the parton densities. The hadron-level cross section at $\mathcal{O}(\alpha)$ can be written as

$$
\begin{aligned}
d \sigma\left(p \vec{p} \rightarrow l^{+} l^{-}+X\right)= & \sum_{a, b=q, \bar{q}, \gamma} \int_{0}^{1} d x_{1} d x_{2} q_{a}\left(x_{1}, M^{2}\right) q_{b}\left(x_{2}, M^{2}\right)\left[d \sigma_{0}^{a b}+d \sigma_{\alpha}^{a b}\right] \\
& -\left(\Delta q_{a}\left(x_{1}, M^{2}\right) q_{b}\left(x_{2}, M^{2}\right)+q_{a}\left(x_{1}, M^{2}\right) \Delta q_{b}\left(x_{2}, M^{2}\right)\right) d \sigma_{0}^{a b}
\end{aligned}
$$

where $a, b$ run over all parton species described by the densities $q_{i}\left(x, M^{2}\right), M$ is the factorization scale, $d \sigma_{0}^{a b}$ and $d \sigma_{\alpha}^{a b}$ are the Born and $\mathcal{O}(\alpha)$ partonic cross sections, initiated by partons $a$ and $b$, respectively. In the case of photon-induced processes, $d \sigma_{0}^{q \gamma}=d \sigma_{0}^{\gamma q}=0 .^{2}$

The relevant $\mathcal{O}(\alpha)$ subtraction terms are

$$
\begin{aligned}
& \Delta q_{i}\left(x, M^{2}\right)=\int_{x}^{1} d z q_{i}\left(\frac{x}{z}, M^{2}\right) \frac{\alpha}{2 \pi} Q_{i}^{2}\left[P_{q \rightarrow q \gamma}(z)\left(\log \left(\frac{M^{2}}{m_{i}^{2}}\right)-2 \log (1-z)-1\right)\right]_{+} \\
&+f_{q}(z)+q_{\gamma}\left(\frac{x}{z}, M^{2}\right) \frac{\alpha}{2 \pi} Q_{i}^{2}\left[P_{\gamma \rightarrow q \bar{q}}(z)\left(\log \left(\frac{M^{2}}{m_{q}^{2}}\right)\right)\right]+f_{\gamma}(z) \\
& \Delta q_{\gamma}\left(x, M^{2}\right)= \sum_{i=q, \bar{q}} \int_{x}^{1} d z q_{i}\left(\frac{x}{z}, M^{2}\right) \frac{\alpha}{2 \pi} Q_{i}^{2}\left[P_{q \rightarrow \gamma q}(z)\left(\log \left(\frac{M^{2}}{m_{i}^{2}}\right)-2 \log (1-z)-1\right)\right]_{+} \\
&+\bar{f}(z)
\end{aligned}
$$

where $Q_{i}$ and $m_{i}$ are the electric charge fraction and the mass of the quark $i$; the functions $f_{i}(z)(i=q, \gamma)$ allow to change the subtraction scheme (e.g. DIS or $\overline{M S}$ ) and are defined as 39

$$
\begin{aligned}
f_{q}(z)= & 2\left(\frac{\log (1-z)}{1-z}\right)_{+}-\frac{3}{2} \frac{1}{(1-z)_{+}}-(1+z) \log (1-z)-\frac{1+z^{2}}{1-z} \log (z) \\
& +3+2 z-\left(\frac{9}{2}+\frac{\pi^{2}}{3}\right) \\
f_{\gamma}(z)= & \left((1-z)^{2}+z^{2}\right) \log \left(\frac{1-z}{z}\right)-8 z^{2}+8 z-1
\end{aligned}
$$

Since also the photon-induced processes contribute to the hadron-level cross section and develope a mass singularity when the outgoing quark is collinear to the incoming photon, also the Altarelli-Parisi splitting function $P_{\gamma \rightarrow q \bar{q}}$ contributes to the subtraction term for the quark densities. The processes $\gamma q \rightarrow l^{+} l^{-} q$, because of a photon exchange in the $t$-channel in the peripheral diagram, develop a collinear singularity, proportional to the splitting function $P_{q \rightarrow \gamma q}(z)$, which can be reabsorbed in the photon density. In the DIS scheme, the function $\bar{f}(z)$ can be fixed by any combination of observables and will be therefore set to zero.

Given the presence in the hadron-level cross section of eq. (3.2) of the product of two parton densities, the subtraction procedure in a factorized form could yield terms of $\mathcal{O}\left(\alpha^{2}\right)$, which have been discarded at $\mathcal{O}(\alpha)$ for consistency.

\footnotetext{
${ }^{2}$ In the numerical evaluation, we omit $\mathcal{O}(\alpha)$ corrections to the subprocess $d \sigma_{0}^{\gamma \gamma}$, because numerically negligible.
} 
The generalization of the independence from the value of the quark masses of the $\mathcal{O}(\alpha)$ cross section of eq. (3.2) to the cross section including also QED higher-order corrections has been discussed in detail in ref. 20] and the main result is our master formula for the computation of the hadron-level cross section and event simulation

$$
\begin{aligned}
d \sigma_{\text {had }}= & \sum_{a, b=q, \bar{q}} \int_{0}^{1} d x_{1} d x_{2} q_{a}\left(x_{1}, M^{2}\right) q_{b}\left(x_{2}, M^{2}\right) \times \\
& \left\{\tilde{F}_{\mathrm{SV}} \tilde{\Pi}\left(Q^{2}, \varepsilon\right) \sum_{n=0}^{\infty} \frac{1}{n !}\left(\prod_{i=0}^{n} \tilde{F}_{H, i}\right)\left|\tilde{\mathcal{M}}_{n, L L}\right|^{2} d \Phi_{n}\right. \\
& \left.+\left[d \sigma_{\alpha}^{a b, s u b}-\left(\frac{\Delta q_{a}\left(x_{1}, M^{2}\right)}{q_{a}\left(x_{1}, M^{2}\right)}+\frac{\Delta q_{b}\left(x_{2}, M^{2}\right)}{q_{b}\left(x_{2}, M^{2}\right)}\right) d \sigma_{0}^{a b}\right]\right\} \\
& +d \sigma_{\text {had }}^{q \gamma}+d \sigma_{\text {had }}^{\gamma \gamma}
\end{aligned}
$$

where $d \sigma_{\text {had }}^{q \gamma}, d \sigma_{\text {had }}^{\gamma \gamma}$ can be derived straightforwardly from eq. (3.2).

The factors with a tilde and also $d \sigma_{\alpha}^{a b, s u b}$ represent quantities subtracted of the initialstate singularities (cfr. section 4 of ref. [20] for the definitions and more details).

\section{Numerical results}

All the numerical results have been obtained using the following values for the input parameters

$$
\begin{array}{lll}
\alpha=1 / 137.03599911 & G_{\mu}=1.1663710^{-5} \mathrm{GeV}^{-2} & m_{Z}=91.1876 \mathrm{GeV} \\
m_{W}=80.398 \mathrm{GeV} & \Gamma_{W}=2.4952 \mathrm{GeV} & m_{H}=115 \mathrm{GeV} \\
m_{e}=510.99892 \mathrm{KeV} & m_{\mu}=105.658369 \mathrm{MeV} & m_{\tau}=1.77699 \mathrm{GeV} \\
m_{u}=66 \mathrm{MeV} & m_{c}=1.2 \mathrm{GeV} & m_{t}=170.9 \mathrm{GeV} \\
m_{d}=66 \mathrm{MeV} & m_{s}=150 \mathrm{MeV} & m_{b}=4.3 \mathrm{MeV} \\
V_{\mathrm{ud}}=0.975 & V_{\mathrm{us}}=0.222 & V_{\mathrm{ub}}=0 \\
V_{\mathrm{cd}}=0.222 & V_{\mathrm{cs}}=0.975 & V_{\mathrm{cb}}=0 \\
V_{\mathrm{td}}=0 & V_{\mathrm{ts}}=0 & V_{\mathrm{tb}}=1
\end{array}
$$

and have been computed in the EW scheme defined in section 2.3. The set of parton density functions used to compute all the hadron-level cross sections is MRST2004QED [27]. In this set of $p d f$ the QCD and the QED factorization scales are set to be equal and, as usually done in the literature [11, 12], we choose $M=m_{Z}$. The use of the $p d f$ set MRST2004QED implies that our numerical results correspond to the DIS factorization scheme. The computation of the hadron-level results requires the numerical evaluation of the subtraction term defined in eq. (3.3); a grid of values in the variable $x$, which is then interpolated, is obtained by means of the numerical library CUBA [40]. All the hadron-level results refer to the LHC, at a nominal c.m. energy $\sqrt{s}=14 \mathrm{TeV}$.

The following cuts have been imposed to select the events

$$
p_{\perp, \ell}>25 \mathrm{GeV}, \quad\left|\eta_{\ell}\right|<2.5
$$



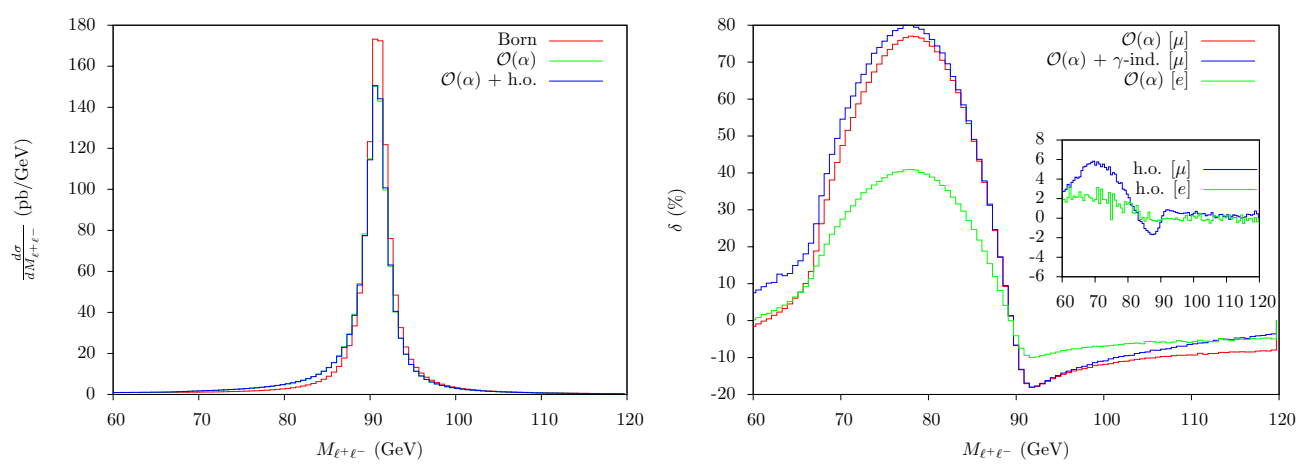

Figure 5: Invariant mass distribution around the $Z$ peak (left) and relative effect of different contributions (right), for bare muons and recombined electrons.
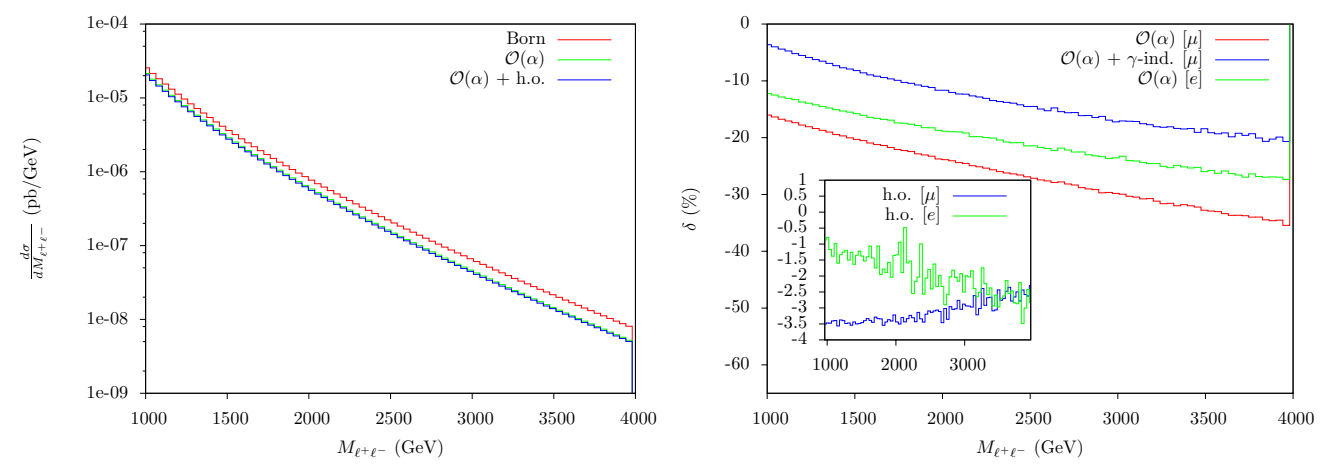

Figure 6: High tail of the invariant mass distribution (left) and relative effect of different contributions (right), for bare muons and recombined electrons.

where $p_{\perp, \ell}$ and $\eta_{\ell}$ are the transverse momentum and the pseudo-rapidity of the charged leptons, respectively.

Our results are obtained for muon pair final states. However, we also show results for recombined electrons in the case of the invariant mass distribution. In fact, we assume perfect isolation of photons from the muon, which is experimentally achievable with good accuracy: the resulting correction is therefore amplified by large muon mass collinear logarithms, because the photon emission is not treated inclusively in the region around the muon. In the case of electrons, it is not possible experimentally to separate them from the photon track, when the latter lies within a cone around the lepton smaller than the detector angular resolution. We adopt the following recombination procedure

- photons with a rapidity $\left|\eta_{\gamma}\right|>2.5$ are never recombined to the electron;

- if the photon rapidity is $\left|\eta_{\gamma}\right|<2.5$ and $R_{e \gamma}=\sqrt{\left(\eta_{e}-\eta_{\gamma}\right)^{2}+\phi_{e \gamma}^{2}}<0.1$ ( $\phi_{e \gamma}$ is the angle between the photon and the electron in the transverse plane), then the photon is recombined with the electron, i.e. the momenta of the two particles are added and associated with the momentum of the electron; 


\begin{tabular}{|l|l|}
\hline 1. & lowest order (Born), as in section 2.3, only $q \bar{q}$ subprocess \\
2. & exact $\mathcal{O}(\alpha)$ EW corrections to the $q \bar{q}$ subprocess \\
3. & exact $\mathcal{O}(\alpha)$ EW corrections to the $q \bar{q}$ subprocess plus $\gamma \gamma$ and $q \gamma$ contributions \\
4. & exact $\mathcal{O}(\alpha)$ EW matched with higher-order QED corrections to the $q \bar{q}$ subprocess \\
\hline
\end{tabular}

Table 1: Different approximations for the calculation of the neutral current Drell-Yan cross section.

\begin{tabular}{|c|c|c|c|c|}
\hline & 1. & 2. & 3. & 4. \\
\hline$\sigma(\mathrm{pb})$ & $739.1(2)$ & $710.7(1)$ & $715.8(1)$ & $712.8(2)$ \\
\hline
\end{tabular}

Table 2: Cross sections, in pb, using approximations 1., 2., 3. and 4. given in table 1 and according to the EW scheme defined in section 2.3.
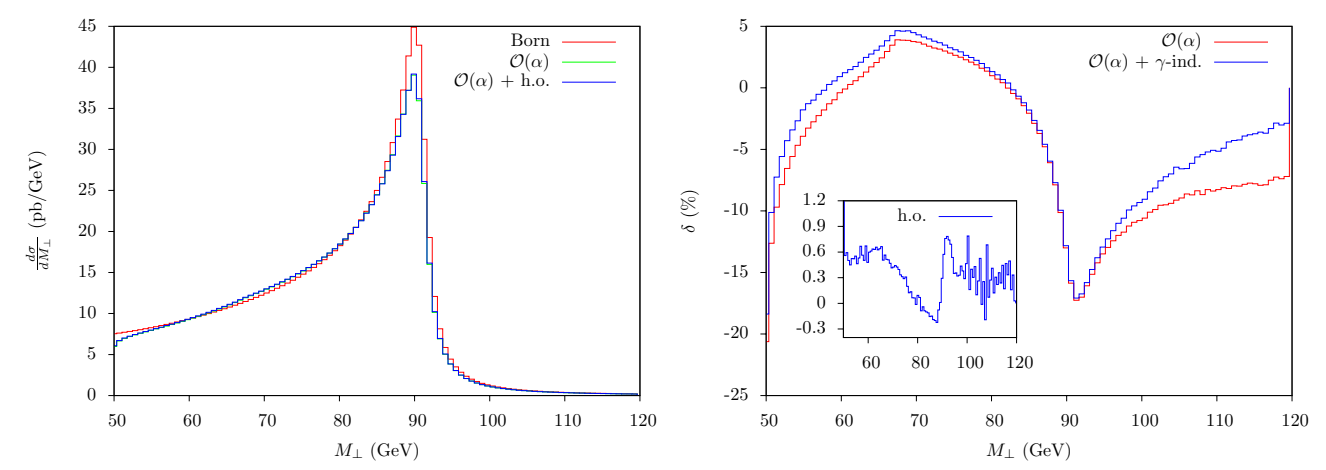

Figure 7: Transverse mass distribution around the $Z$ peak (left) and relative effect of different contributions (right).

- the resulting momenta should satisfy the cuts of eq. (4.1).

In order to study the different effects of the radiative corrections and partonic subprocesses on the relevant observables, we will distinguish the approximations described in table 目.

In table 2 we compare the values of the total cross section, within the cuts specified above, calculated in the approximations 1., 2., 3. and 4. of table 1. The effect of the $\mathcal{O}(\alpha)$ corrections is negative, of about $4 \%$ of the lowest-order result, while photonic subprocesses and QED higher orders enhance the $\mathcal{O}(\alpha)$ cross section of about $1 \%$ and a few per mille, respectively. In figure 5 we show the invariant mass distribution of the final-state lepton pair, which is peaked at the $Z$-boson mass value and which, taking as a reference the high-precision LEP measurement, can be used to calibrate the LHC detectors. The radiative corrections significantly modify the shape of this distribution, as can be seen from the right panel of figure 5, showing the relative effect, expressed in units of the Born cross section (approximation 1.), of the different contributions corresponding to approximations 2., 3. and 4. of table 1. There is a well-known effect [11, 19] due to final-state photon radiation which increases, in the muon pair final state, by almost $80 \%$ the lowest-order result in the region below the $Z$ peak. Around the peak, instead, the correction is negative of about 

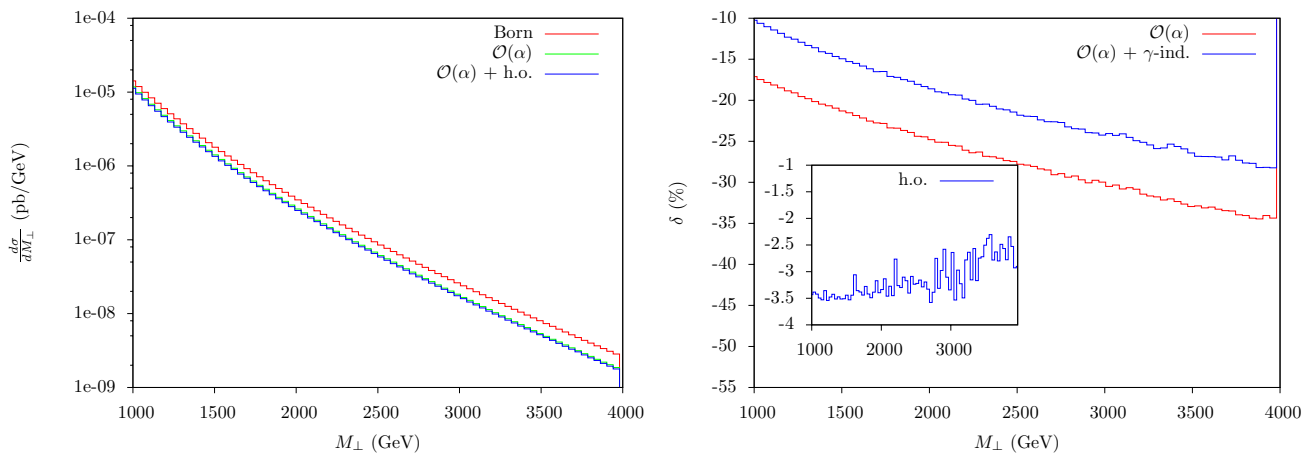

Figure 8: High tail of the transverse mass distribution (left) and relative effect of different contributions (right).
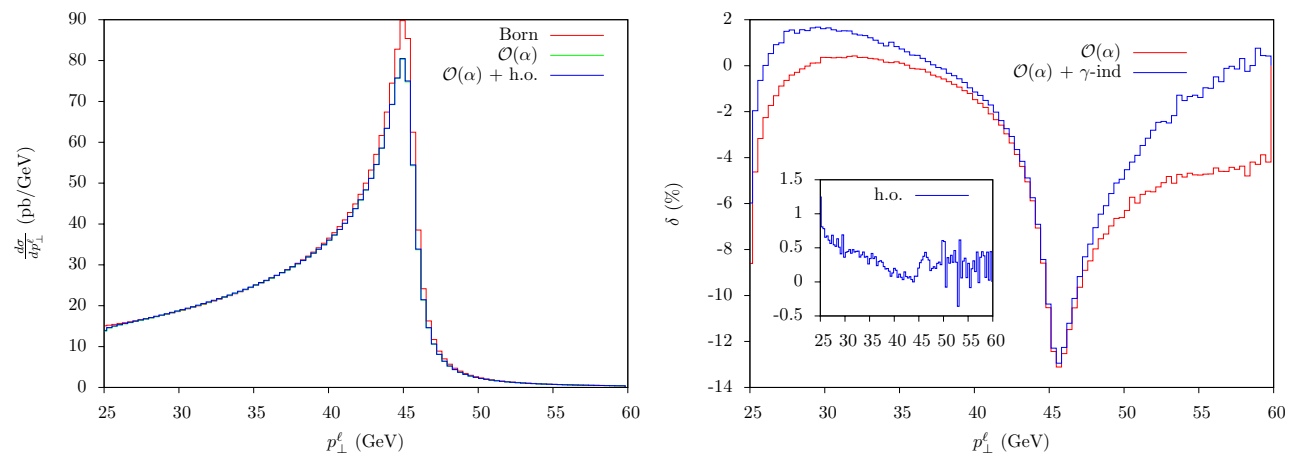

Figure 9: Lepton transverse momentum distribution around the $Z$ peak (left) and relative effect of different contributions (right).
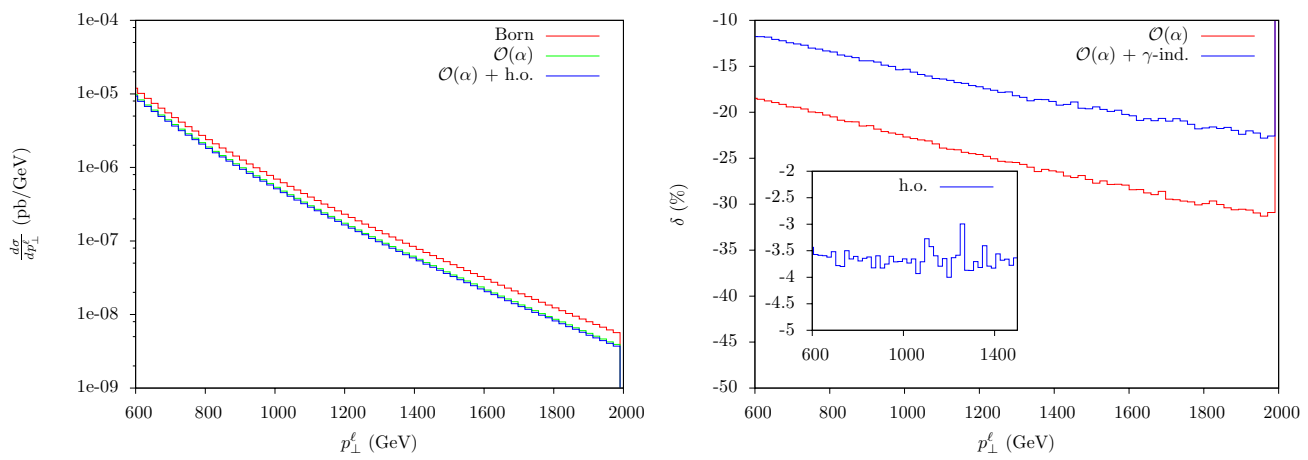

Figure 10: High tail of the lepton transverse momentum distribution (left) and relative effect of different contributions (right).

$-15 \%$. The size of these corrections is significantly reduced when considering electron final states, in agreement with the results known in the literature [11] and due to the photon recombination procedure, which implies a partial cancelation of the mass logarithms, according to the KLN theorem. The impact of the photon-induced processes is of the order 

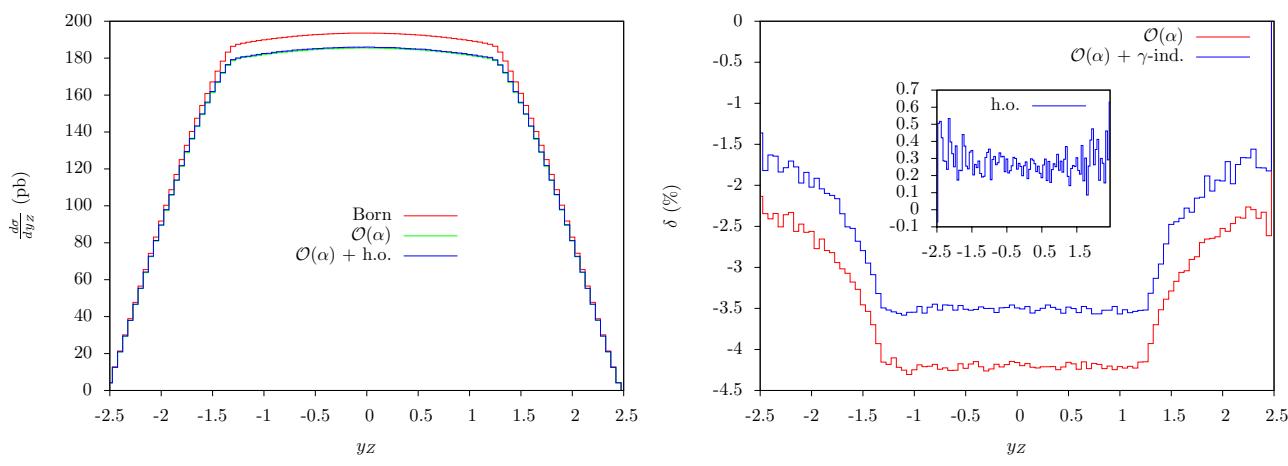

Figure 11: Rapidity distribution of the $Z$ boson (left) and relative effect of different contributions (right).
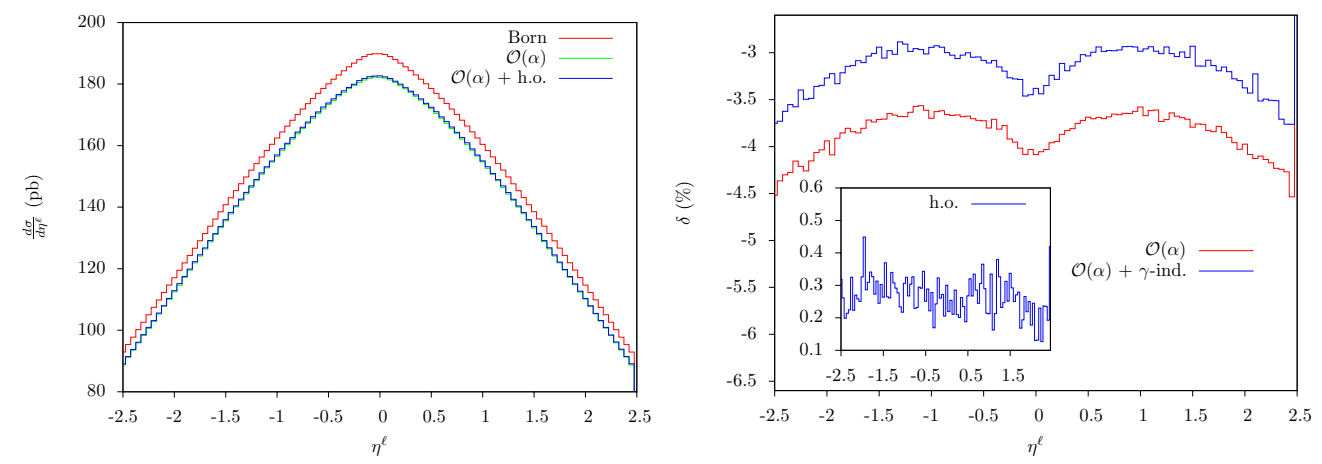

Figure 12: Pseudo-rapidity distribution of the final state lepton (left) and relative effect of different contributions (right).

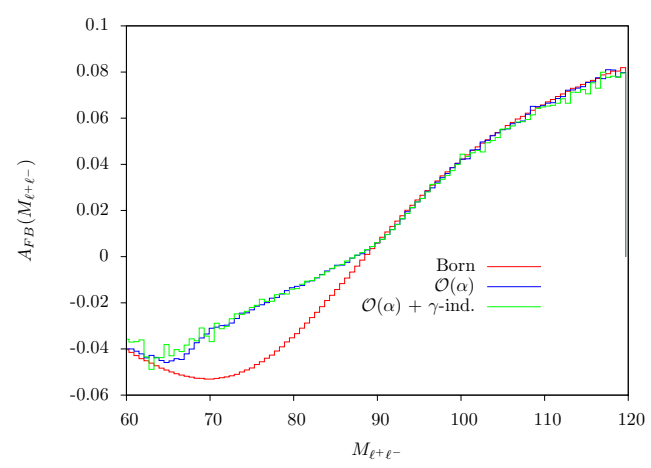

Figure 13: Forward-Backward asymmetry as a function of the invariant mass of the lepton pair according to the approximations 1., 2. and 3. of table 1.

of a few per cent and is particularly evident away from the resonance region. The effect of multiple photon emission amounts to a few per cent (see the inset of the figure) and can not be neglected for an accurate detector calibration and precision $Z$-physics studies at hadron colliders, as already remarked in ref. [19]. The $Z$-boson mass shift induced by 
QED higher order corrections in fits to the invariant mass distribution has been already quantified in ref. [19] and found to be about $10 \%$ and of opposite sign of that caused by one-photon emission.

In figure 6 we show the invariant mass distribution in the high tail region, where the Drell-Yan processes represent an important background to the searches for new heavy gauge bosons. The distribution receives large negative radiative corrections, of the order of a few ten per cent, due to EW Sudakov logarithm, as previously emphasized in refs. [12, 13. ${ }^{3}$ With electron final states, the photon recombination procedure reduces the negative effect of the $\mathcal{O}(\alpha)$ corrections. The multiple photon emission yields a few per cent effect in this invariant mass region, while the photon-induced processes raise the cross section by approximately $+12 \%$ of the Born approximation. The $\gamma \gamma \rightarrow l^{+} l^{-}$subprocess contributes with about $+3 \%$ of the $q \bar{q}$ Born cross section. Actually, its contribution is suppressed by the smallness of the photon density and can be mainly observed at large invariant masses of the lepton pair.

In figure 7 we consider the distribution of the $Z$ transverse mass, defined as

$$
M_{\perp}=\sqrt{2 p_{\perp, l^{+}} p_{\perp, l^{-}}\left(1-\cos \phi_{l^{+} l^{-}}\right)}
$$

where $\phi_{l^{+} l^{-}}$is the angle between the leptons in the transverse plane. We remark the different size of the effect of the $\mathcal{O}(\alpha)$ corrections, with respect to the invariant mass distribution, to this quantity, that can be useful, in association with the $W$ transverse mass distribution, to measure the $W$-boson mass. In the vicinity of the $Z$ resonance, the relative contribution of $\mathcal{O}(\alpha)$ EW corrections amounts to $\sim-17 \%$ and is about a factor of two of the corresponding effect on the $W$ transverse mass distribution in the charged current channel [20, 38]. This can be simply understood in terms of the dominance of finalstate QED radiation within the full set of $\mathcal{O}(\alpha)$ EW corrections around the $Z$ resonance and, consequently, because of the presence of two radiating leptons in the neutral current channel. Photon-induced processes reduce the relative size of $\mathcal{O}(\alpha)$ EW corrections and give a contribution of some per cent, especially above the $Z$ peak, while multiple photon corrections contribute at some per mille level.

In figure 8 we show the high tail of the transverse mass distribution, which also represents an important background observable to the searches for new neutral gauge bosons. The presence of the EW Sudakov logarithms reduces the cross section by 18 to $35 \%$ for $1 \mathrm{TeV} \leq M_{\perp} \leq 4 \mathrm{TeV}$. In contrast to the invariant mass case, the contribution of the photon induced processes is smaller in this case, of the order of $+7 \%$, while the effect of higher-order QED corrections is of the same size as that observed in the high tail of the invariant mass distribution.

In figure 9 the lepton transverse momentum distribution is shown around the $Z$ peak and, in figure 10, in the high momentum tail. The effect of the radiative corrections, as well as of photon-induced processes, shows a pattern similar to the one observed for the transverse mass distribution and discussed above.

\footnotetext{
${ }^{3}$ It has been recently noticed that weak boson emission diagrams, that contribute at the same order of $\mathcal{O}(\alpha)$ EW corrections, lead to a partial cancellation of EW Sudakov corrections, when the weak boson decays into unobserved $\nu \bar{\nu}$ or jet pairs 41 .
} 
The rapidity distribution of the $Z$ boson is presented in figure 11. The $\mathcal{O}(\alpha)$ EW corrections are negative, of the order of $-4 \%$, and almost constant in the interval $\left|y_{Z}\right| \leq 1$, and are smaller in size, at the $2 \%$ level, for larger values of the rapidity. It is worth noting that $\mathcal{O}(\alpha)$ EW contributions to such an observables are of the same order of magnitude as NNLO QCD corrections [10] and, therefore, both the effects need to be taken into account in precision measurements of the $Z$ rapidity distribution. We notice the overall positive correction due the photon-induced processes, at the $1 \%$ level. The multiple photon emission has an almost negligible impact on this observable, at the per mille level, as already discussed in ref. [19].

The numerical results for the pseudo-rapidity of the final state lepton and the relative effect of the radiative corrections and photon-induced processes on this distribution are illustrated in figure 12, showing a pattern quite similar to that observed for the $Z$ rapidity.

At hadron colliders it is possible to define a forward-backward asymmetry $A_{\mathrm{FB}}$ and to derive from it a measurement of the leptonic effective weak mixing angle $\sin ^{2} \theta_{\text {eff }}^{l}$. The forward-backward asymmetry can be written as

$$
\begin{aligned}
A_{\mathrm{FB}}\left(M_{l^{+} l^{-}}\right) & =\frac{F\left(M_{l^{+} l^{-}}\right)-B\left(M_{l^{+} l^{-}}\right)}{F\left(M_{l^{+} l^{-}}\right)+B\left(M_{l^{+} l^{-}}\right)} \\
F\left(M_{l^{+} l^{-}}\right) & =\int_{0}^{1} d \cos \theta^{*} \frac{d \sigma}{d \cos \theta^{*}} \quad B\left(M_{l^{+} l^{-}}\right)=\int_{-1}^{0} d \cos \theta^{*} \frac{d \sigma}{d \cos \theta^{*}}
\end{aligned}
$$

where

$$
\begin{aligned}
\cos \theta^{*} & =f \frac{2}{M\left(l^{+} l^{-}\right) \sqrt{M^{2}\left(l^{+} l^{-}\right)+p_{\perp}^{2}\left(l^{+} l^{-}\right)}}\left[p^{+}\left(l^{-}\right) p^{-}\left(l^{+}\right)-p^{+}\left(l^{+}\right) p^{-}\left(l^{-}\right)\right] \\
p^{ \pm} & =\frac{1}{\sqrt{2}}\left(E \pm p_{z}\right), \quad f=1 \text { (Tevatron), } \quad f=\frac{\left|p_{z}\left(l^{+} l^{-}\right)\right|}{p_{z}\left(l^{+} l^{-}\right)}(\mathrm{LHC})
\end{aligned}
$$

and $M\left(l^{+} l^{-}\right)$is the invariant mass of the final-state lepton pair, $p_{\perp}\left(l^{+} l^{-}\right)$and $p_{z}\left(l^{+} l^{-}\right)$ are the total transverse momentum and total longitudinal momentum of the $l^{+} l^{-}$pair, respectively. The asymmetry can be expressed in terms of $\sin ^{2} \theta_{\text {eff }}^{l}$, with good approximation, as $A_{\mathrm{FB}}=b\left(a-\sin ^{2} \theta_{\text {eff }}^{l}\right)$. A detailed description of the effect of the $\mathcal{O}(\alpha)$ corrections on the coefficients $a, b$ can be found in ref. [12], together with an analysis of the relevant backgrounds. In figure 13 we present the asymmetry distribution, evaluated with the cuts of eq. (4.1), according to the approximations 1., 2. and 3. of table 1. It can be seen that $\mathcal{O}(\alpha)$ EW corrections are relevant and modify the shape of $A_{\mathrm{FB}}$ below the $Z$ peak, whereas the photon-induced processes, as for the multiple photon corrections [19], do not contribute significantly to this observable.

\section{Conclusions}

In this paper we have presented a precision electroweak calculation of the neutral current Drell-Yan process. The theoretical approach is based on the matching of exact $\mathcal{O}(\alpha) \mathrm{EW}$ corrections with QED Parton Shower, to account for the effect of multiple photon emission. The use of the proton $p d f$ parametrization MRST2004QED allows for the presence of a photon 
density in the proton and gives rise to additional partonic subprocesses which contribute to the inclusive Drell-Yan signature. The impact of the different contributions studied in the paper turns out to be important, in association with higher-order QCD effects, in view of the precision measurements of EW parameters at the Fermilab Tevatron and the CERN LHC, as well as to validate the existing $p d f$ parametrizations and to assess the SM normalization in the search for new neutral heavy gauge bosons.

The calculation has been carried out using as inputs $\alpha(0), m_{W}, m_{Z}$ rather than a LEPlike choice $\alpha(0), G_{\mu}, m_{Z}$, in order to keep both gauge boson masses as free parameters which can be fitted from the data, since $W$ - and $Z$-boson physics are intimately related at hadron colliders.

Possible perspectives of the present paper include tuned comparisons with independent calculations, in the spirit of the work done for single $W$ production during the Les Houches 2005 [42] and the TeV4LHC [43] workshops, a combination of EW and QCD corrections at the event generator level and the addition of higher-order contributions such as 2-loop EW Sudakov logarithms 44].

\section{Acknowledgments}

We are grateful to Andreij Arbuzov and Dima Bardin for the numerical comparisons at the preliminary stage of this work. C.M.C.C. is supported by a "Angelo Della Riccia" fellowship and thanks the CERN for the hospitality. He also thanks the Royal Society and the British Council for partial support and is grateful to the School of Astronomy and Physics of the Southampton University and the NExT Institute where part of this work was done. A.V. is supported by the European Community's Marie-Curie Research Training Network under contract MRTN-CT-2006-035505 (HEPTOOLS).

\section{References}

[1] S.D. Drell and T.-M. Yan, Massive lepton pair production in hadron-hadron collisions at high-energies, Phys. Rev. Lett. 25 (1970) 316 [Erratum ibid. 25 (1970) 902].

[2] CDF collaboration, D.E. Acosta et al., Measurement of the forward-backward charge asymmetry of electron positron pairs in p p collisions at $\sqrt{s}=1.96$ TeV, Phys. Rev. D 71 (2005) 052002 hep-ex/0411059;

ATLAS collaboration, M. Aharrouche, Forward-backward charge asymmetry in $Z$ production at the $L H C$, arXiv:0705.3757.

[3] CDF collaboration, V.M. Abazov et al., Combination of CDF and D0 results on $W$ boson mass and width, Phys. Rev. D 70 (2004) 092008 hep-ex/0311039;

CDF collaboration, A. Abulencia et al., Measurements of inclusive $W$ and $Z$ cross-sections in $p \bar{p}$ collisions at $\sqrt{s}=1.96 \mathrm{TeV}$, hep-ex/0508029;

CDF collaboration, T. Aaltonen et al., First run II measurement of the $W$ boson mass, arXiv:0708.3642.

[4] H.L. Lai et al., Global QCD analysis and the Cteq parton distributions, Phys. Rev. D 51 (1995) 4763 hep-ph/9410404; 
A.D. Martin, R.G. Roberts, W.J. Stirling and R.S. Thorne, Uncertainties of predictions from parton distributions. I: experimental errors., Eur. Phys. J. C 28 (2003) 455 hep-ph/0211080.

[5] M. Dittmar, F. Pauss and D. Zurcher, Towards a precise parton luminosity determination at the CERN LHC, Phys. Rev. D 56 (1997) 7284 hep-ex/9705004.

[6] S. Frixione and M.L. Mangano, How accurately can we measure the $W$ cross section?, JHEP 05 (2004) 056 hep-ph/0405130.

[7] CDF collaboration, F. Abe et al., Search for new gauge bosons decaying into dileptons in $\bar{p} p$ collisions at $\sqrt{s}=1.8$ TeV, Phys. Rev. Lett. 79 (1997) 2192.

[8] G. Altarelli, R.K. Ellis and G. Martinelli, Large perturbative corrections to the Drell-Yan process in QCD, Nucl. Phys. B 157 (1979) 461.

[9] R. Hamberg, W.L. van Neerven and T. Matsuura, A complete calculation of the order $\alpha_{S}^{2}$ correction to the Drell-Yan K factor, Nucl. Phys. B 359 (1991) 343 [Erratum ibid. B 644 (2002) 403].

[10] C. Anastasiou, L.J. Dixon, K. Melnikov and F. Petriello, Dilepton rapidity distribution in the Drell-Yan process at NNLO in QCD, Phys. Rev. Lett. 91 (2003) 182002 hep-ph/0306192;

C. Anastasiou, L.J. Dixon, K. Melnikov and F. Petriello, High-precision QCD at hadron colliders: electroweak gauge boson rapidity distributions at NNLO, Phys. Rev. D 69 (2004) 094008 hep-ph/0312266;

K. Melnikov and F. Petriello, Electroweak gauge boson production at hadron colliders through $O\left(\alpha_{s}^{2}\right)$, Phys. Rev. D 74 (2006) 114017 hep-ph/0609070.

[11] U. Baur, S. Keller and W.K. Sakumoto, QED radiative corrections to Z boson production and the forward backward asymmetry at hadron colliders, Phys. Rev. D 57 (1998) 199 hep-ph/9707301.

[12] U. Baur, O. Brein, W. Hollik, C. Schappacher and D. Wackeroth, Electroweak radiative corrections to neutral-current Drell-Yan processes at hadron colliders, Phys. Rev. D 65 (2002) 033007 hep-ph/0108274.

[13] V.A. Zykunov, Weak radiative corrections to the Drell-Yan process for large invariant mass of a dilepton pair, Phys. Rev. D 75 (2007) 073019 hep-ph/0509315.

[14] G. Corcella et al., HERWIG 6.5 release note, hep-ph/0210213.

[15] T. Sjostrand et al., High-energy-physics event generation with PYTHIA 6.1, Comput. Phys. Commun. 135 (2001) 238 hep-ph/0010017.

[16] S. Frixione and B.R. Webber, Matching NLO QCD computations and parton shower simulations, JHEP 06 (2002) 029 hep-ph/0204244.

[17] C. Balázs and C.P. Yuan, Soft gluon effects on lepton pairs at hadron colliders, Phys. Rev. D 56 (1997) 5558 hep-ph/9704258.

[18] C.M. Carloni Calame, G. Montagna, O. Nicrosini and M. Treccani, Higher-order QED corrections to $W$-boson mass determination at hadron colliders, Phys. Rev. D 69 (2004) 037301 hep-ph/0303102.

[19] C.M. Carloni Calame, G. Montagna, O. Nicrosini and M. Treccani, Multiple photon corrections to the neutral-current Drell-Yan process, JHEP 05 (2005) 019 hep-ph/0502218. 
[20] C.M. Carloni Calame, G. Montagna, O. Nicrosini and A. Vicini, Precision electroweak calculation of the charged current Drell-Yan process, JHEP 12 (2006) 016 hep-ph/0609170.

[21] C.M. Carloni Calame, C. Lunardini, G. Montagna, O. Nicrosini and F. Piccinini, Large-angle Bhabha scattering and luminosity at flavour factories, Nucl. Phys. B 584 (2000) 459 hep-ph/0003268;

C.M. Carloni Calame, An improved parton shower algorithm in QED, Phys. Lett. B 520 (2001) 16 hep-ph/0103117.

[22] P. Golonka and Z. Was, Photos Monte Carlo: a precision tool for QED corrections in Z and $W$ decays, Eur. Phys. J. C 45 (2006) 97 hep-ph/0506026.

[23] W.T. Giele and S. Keller, Determination of $W$ boson properties at hadron colliders, Phys. Rev. D 57 (1998) 4433 hep-ph/9704419.

[24] C.M. Carloni Calame, talk given at CMS meeting, CERN (2007);

G. Montagna, talk given at the EPS HEP2007 Conference, Manchester U.K. (2007).

[25] Z. Nagy and D.E. Soper, QCD and Monte Carlo event generators, hep-ph/0607046; P.Z. Skands, QCD (and) event generators, AIP Conf. Proc. 792 (2005) 73 hep-ph/0507129.

[26] G. Balossini, C.M. Carloni Calame, G. Montagna, O. Nicrosini and F. Piccinini, Matching perturbative and parton shower corrections to Bhabha process at flavour factories, Nucl. Phys. B 758 (2006) 227 hep-ph/0607181.

[27] A.D. Martin, R.G. Roberts, W.J. Stirling and R.S. Thorne, Parton distributions incorporating QED contributions, Eur. Phys. J. C 39 (2005) 155 hep-ph/0411040.

[28] A.B. Arbuzov and R.R. Sadykov, Inverse bremsstrahlung contributions to Drell-Yan like processes, arXiv:0707.0423.

[29] T. Hahn, Generating Feynman diagrams and amplitudes with FeynArts 3, Comput. Phys. Commun. 140 (2001) 418 hep-ph/0012260.

[30] T. Hahn and M. Pérez-Victoria, Automatized one-loop calculations in four and d dimensions, Comput. Phys. Commun. 118 (1999) 153 hep-ph/9807565.

[31] G.J. van Oldenborgh and J.A.M. Vermaseren, New algorithms for one loop integrals, Z. Physik C 46 (1990) 425.

[32] L.F. Abbott, The background field method beyond one loop, Nucl. Phys. B 185 (1981) 189; A. Denner, G. Weiglein and S. Dittmaier, Application of the background field method to the electroweak standard model, Nucl. Phys. B 440 (1995) 95 hep-ph/9410338.

[33] F. Jegerlehner, Hadronic contributions to the photon vacuum polarization and their role in precision physics, J. Phys. G 29 (2003) 101 hep-ph/0104304.

[34] A. Sirlin, Radiative corrections in the $\mathrm{SU}(2)_{l} \times \mathrm{U}(1)$ theory: a simple renormalization framework, Phys. Rev. D 22 (1980) 971.

[35] A. Denner, Techniques for calculation of electroweak radiative corrections at the one loop level and results for $W$ physics at LEP-200, Fortschr. Phys. 41 (1993) 307 arXiv:0709.1075.

[36] G. Degrassi and A. Sirlin, Comparative analysis of electroweak corrections to $e^{+} e^{-} \rightarrow F \bar{F}$ in on-shell and $\overline{M S}$ frameworks, Nucl. Phys. B 352 (1991) 342.

[37] U. Baur, S. Keller and D. Wackeroth, Electroweak radiative corrections to $W$ boson production in hadronic collisions, Phys. Rev. D 59 (1999) 013002 hep-ph/9807417. 
[38] S. Dittmaier and M. Kramer, Electroweak radiative corrections to $W$-boson production at hadron colliders, Phys. Rev. D 65 (2002) 073007 hep-ph/0109062.

[39] K.P.O. Diener, S. Dittmaier and W. Hollik, Electroweak radiative corrections to deep-inelastic neutrino scattering: implications for NuTeV?, Phys. Rev. D 69 (2004) 073005 hep-ph/0310364.

[40] T. Hahn, CUBA: a library for multidimensional numerical integration, Comput. Phys. Commun. 168 (2005) 78 hep-ph/0404043.

[41] U. Baur, Weak boson emission in hadron collider processes, Phys. Rev. D 75 (2007) 013005 hep-ph/0611241.

[42] C. Buttar et al., Les Houches physics at TeV colliders 2005, standard model, QCD, EW and Higgs working group: summary report, hep-ph/0604120.

[43] TeV4LhC-Top and Electroweak Working Group collaboration, C.E. Gerber et al., Tevatron-for-LHC report: top and electroweak physics, arXiv:0705.3251.

[44] B. Jantzen, J.H. Kühn, A.A. Penin and V.A. Smirnov, Two-loop electroweak logarithms in four-fermion processes at high energy, Nucl. Phys. B 731 (2005) 188 [Erratum ibid. B 752 (2006) 327] hep-ph/0509157;

A. Denner, B. Jantzen and S. Pozzorini, Two-loop electroweak next-to-leading logarithmic corrections to massless fermionic processes, Nucl. Phys. B 761 (2007) 1 hep-ph/0608326. 